\title{
Sleep Apnea and Heart Disease
}

Tyler Baillargeon

Plan B paper

Clinical Exercise Physiology

University of Wisconsin-River Falls

January 15, 2016

Major Advisor: Dr. Joseph O'Kroy

Date: 


\section{$\underline{\text { Sleep Apnea and Heart Disease }}$}

Heart disease (HD) is killing almost 600,000 people annually. This life threatening phenomenon is more common in men than in women and is the leading cause of death for both genders (Center for Disease Control and Prevention, 2014). Heart disease represents a number of problems with the heart (some of which can be linked to heart failure (HF)). Research is now finding a strong correlation between sleep HD, HF and sleep apnea (SA). It is important to understand that HF and HD can lead to serious issues such as heart attacks and even death. Therefore, there are potentially a great deal of heart problems strongly linked to SA. The most serious of the issues associated with SA is a myocardial infarction. This paper will explore the relationship between SA and multiple forms of HD.

The word apnea is Greek and means "without breath." SA is when an individual involuntarily stops breathing in their sleep. SA is also known as sleep disordered breathing (SDB). There are a multitude of common SA risk factors including daytime sleepiness, snoring, large neck circumference, obesity, and high BMI values. Since a large portion of United States citizens struggle with obesity, large neck circumferences, and high BMI values, the American public is very susceptible to SA and HD.

There are three types of SA including obstructive, central, and mixed. Obstructive SA (OSA) is more of a peripheral or mechanical issue that is caused by a blockage of the airway. It typically occurs when the soft tissue in the rear of the throat collapses while one is sleeping. The airway is not blocked at all with central SA (CSA), but rather the brain fails to signal the muscles to breathe. The last type of SA is known 
as mixed SA (MSA) and it not actually a third form, but rather a combination of obstructive and central SA. The combination of obstructive and central SA is also referred to as complex (compSAS). According to the National Sleep Foundation (2005), more than 18 million Americans are affected by SA. It is important that more research is conducted and that the population becomes educated due to the severe impact SA has on heart health.

Over the years, increasing numbers of people are diagnosed with SA to treat some of the commonly associated symptoms. According to the National Heart, Lung, and Blood Institute $(\mathrm{NIH})$ some of these symptoms are being tired during the awake hours (daytime sleepiness), loud snoring, restless sleep, and waking up with a choking or gasping sensation. One common treatment method for SA is the use of a Continuous Positive Airway Pressure (CPAP) machine. A CPAP machine involves a nasal or face mask connected to a pump, providing positive air pressure to prevent airway obstruction. There is minimal public knowledge regarding the positive impact a CPAP machine has to treat sleep apnea and how it can positively impact the heart's function. As a result of the increasing number of HF problems and SA risk factors, it is imperative that individuals are properly informed of the impact SA has on the heart as well as the benefits of CPAP treatment. 


\section{Sleep Apnea (Sleep Disordered Breathing)}

Aronson et al. (2014) found that of those admitted to the hospital for an acute myocardial infarction (AMI), a large number of these patients had undiagnosed SA. Aside from an increased left atrial (LA) dimension, there were no other structural or functional changes in the hearts formation. It was also noted that there was a moderate correlation between LA size and oxygen desaturation index (ODI). they did find that pulmonary artery systolic pressure was elevated in the patients with SA [4].

Aronson et al. did find that SA was not correlated with any noticeable impact on clinical outcomes aside from the potential to have HF. Out of 100 patients that were enrolled for an acute myocardial infarction (AMI), 64\% were found to have SA (study defined SA as having more than 5 oxygen desaturation index (ODI) events an hour) [4]. Although methods of diagnosing SA and definitions of SA differ, it has been consistently found that there is a high prevalence of SA (22\%-69\%) in patients with acute coronary syndrome. Patients with stable coronary heart disease have a prevalence of SA somewhere between $30 \%$ and $50 \%$.

A study of 1,210 consecutive subjects found that severe SA (those with an Apnea Hypopnea Index $\mathrm{AHI}>30$ ) was present in over $65 \%$ of the participants. In this same population, over $25 \%$ of the participants had moderate to mild SA (AHI 5-30). This left just under $9 \%$ of the population with no SA $(\mathrm{AHI}<5)$ [2]. Patients with severe SA were typically older, male, and have a higher BMI. Patients with a higher CHADS2 score were also more likely to have severe SA. This correlation has been reinforced by other studies that demonstrate a reduction in atrial fibrillation (AF) when using CPAP 
treatment for SA [6]. This is suggesting that with the use of CPAP some individuals may be able to reduce problems with AF.

Nieto and Peppard et. al. found that SA is strongly associated with metabolic syndrome. Similar to other reports, there was a correlation between SA, diabetes and insulin resistance. There is an association with a delayed decline in glucose following a glucose injection and decreased insulin sensitivity. In other words, the findings suggested that SA escalated diabetes in individuals that had both SA and diabetes concurrently. Diabetes or SA independently is problematic and can lead to many undesirable side effects. That said, it is very important for individuals to be properly treated for both of these issues to avoid additional complications.

Nieto and Young et. al found that there might be an association between SA and hypertension. This association was found to be particularly true for middle-aged participants. More prospective studies regarding the association between SA, blood pressure and hypertension will be helpful in explaining the true magnitude of the association. If this association is found to be true, it is very important to further investigate. Hypertension can be a very serious problem especially if it is not properly treated or diagnosed. The findings indicating that SA might independently affect hypertension is of high concern. This is due to the fact that hypertension can lead to many complications. According to the American Heart Association (AHA) some of these complications include stroke, coronary artery disease, artery damaging and narrowing, kidney failure and many more complications. If further research confirms these findings, properly diagnosing and treating SA will become even more important. 


\section{Peripheral and Mechanical SA}

OSA is one of the most common respiratory disorders in humans. OSA is when an individual's airway is obstructed as displayed in the image below. In this situation, an individual has repetitive pauses in breathing while sleeping despite efforts to breathe. There is more evidence emerging linking OSA to vascular disease and more specifically hypertension. A relatively common disorder, it is estimated that $4 \%$ of males and $2 \%$ of females suffer from OSA [1]. Regular snoring, disturbances in sleep quality, and cognitive dysfunction are found to be associated with SA. One of the main problems associated with having OSA is the effect it has on awake time. One of the main symptoms of OSA is being excessively tired during the day. Individuals with OSA experience excessive tiredness even when sleeping reasonable quantities each day.

(Image 1)

\section{Normal breathing during sleep}

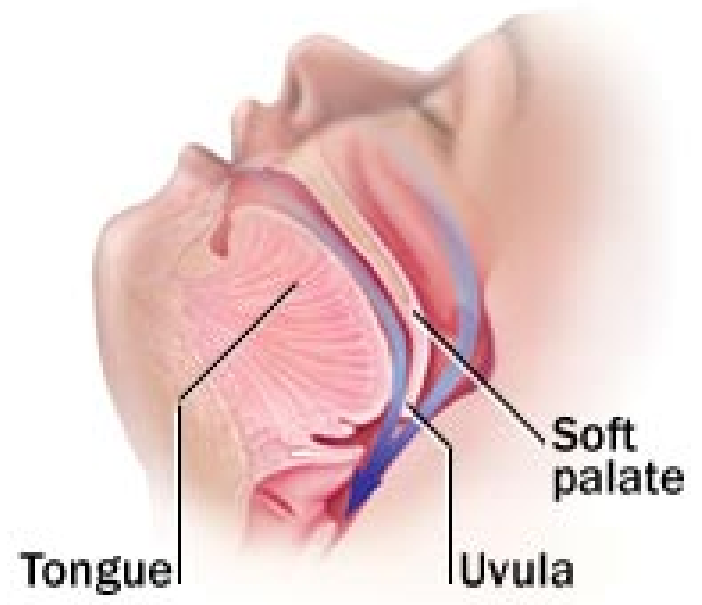

Obstructive sleep apnea

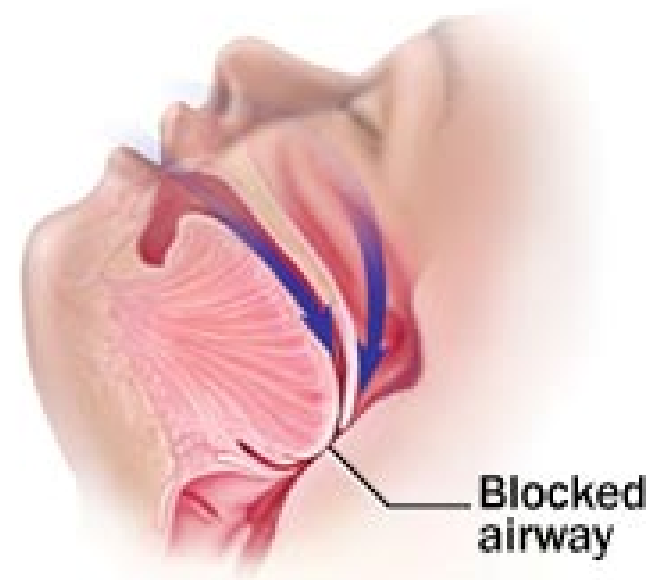

\section{Q Mayo Foundation for Medical Education and Research. All rights reserved.}


Kendzerska, Gershon, Hawker, Leung, and Tomlinson (2014) found that the strongest OSA-related predictor of cardiovascular events or all-cause mortality was the total amount of time that one spent sleeping with an oxygen saturation below $90 \%$. This also indicated that there was a $50 \%$ increased chance or risk of a cardiovascular event or death [1]. Some of the other statistically significant risk factors include total sleep quantity, daytime sleepiness, number of awakenings, amongst others. This finding suggests that testing oxygen saturation during sleep would be a relatively easy way to determine the prevalence and severity of SA. This information is helpful when diagnosing patients and referring for more strenuous testing to determine other potential impacts and negative outcomes.

Javadi, Jalilolghadr, Yazdi, and Majd (2014) found that a high prevalence of OSA was reported in individuals that were scheduled to have an angiography completed. Studies have shown that SA (even without other risk factors) can be a risk factor for cardiovascular disease. People with SA have a roughly $100 \%$ increased risk for cardiovascular disease compared to individuals without SA [11]. It was also found that those patients with OSA had more intense or severe coronary artery disease than those with mild to no OSA.

Javadi, Jalilolghadr, et. al. studied 845 patients between the ages of 40 to 70 scheduled for a coronary angiography. The study exposed a correlation between SA and $\mathrm{HD}$. Of the 845 subjects, $52.2 \%$ were put in the high risk category and the rest in the low risk group for apnea. In addition, $45.8 \%$ of the subjects reported experiencing excessive daytime sleepiness in an ESS questionnaire [11]. Of the subjects, a higher prevalence of OSA was reported. Of the subjects with daytime sleepiness, OSA was 
more intense with more intense sleepiness. Although the reason for correlation is not the most well-known, evidence still shows that breathing disorders during sleep can result in high systolic blood pressure. Patients with coronary stenosis showed a high correlation with OSA. $80 \%$ of patients with OSA were found to have narrowing in at least two of their coronary vessels [6]. The study also showed a high prevalence of OSA in patients scheduled for an angiogram. The findings of this study validated that patients with OSA have more intense artery disease compared to those without OSA. Coronary stenosis is correlated with OSA and signs of OSA were common in these patients. The information in this study further justifies the correlation and need to better understand OSA. Narrowing of the vessels or coronary stenosis are major problems concerning the proper function of the cardiovascular system as a whole.

The study also found that $39 \%$ of patients with single coronary artery stenosis had a high risk of having OSA. About $62 \%$ of patients with 2 or 3 vessel stenosis were classified as a high risk group for OSA [11]. Another area that was studied was the relationship between characteristics of an ECG and factors filled out on the Berlin Questionnaire. $60 \%$ of the patients with evidence of ischemia on their ECG were categorized as high risk for having OSA along with $81 \%$ that had shown signs for a previous myocardial infarction shown by a pathologic Q wave [11]. Individuals at high risk for OSA had lower levels of high-density lipoproteins (HDL) and cholesterol, and higher level of triglycerides. High levels of triglycerides may independently suggest that the individuals would have low HDL and high low-density lipoproteins (LDL). Overall, this study found that OSA can be a risk factor for HD. In addition, screening for OSA 
can be very beneficial in patients that knowingly have HD. If SA is treated early it can prevent the high morbidity rate as well as mortality rate of HD.

In a study by Javadi et. al. they used a model including age, sex, BMI, smoking, neck circumference, waist circumference, triglyceride, HDL cholesterol, fasting blood sugar (FBS), daytime sleepiness, and number of vessels with stenosis according to the angiograph [11]. This study shows that gender and BMI were not statistically associated with having OSA. Sex, FBS, daytime sleepiness, and number of vessels containing stenosis detected with angiography were the variables with the highest OR's related to the presence of OSA in an individual [11].

One study conducted by Chen, Su et. al. on OSA sought to investigate the impact of OSA on cardiac function and remodeling in patients without congestive HF. The researchers first screened patients for SA and then enrolled individuals with SA into the study. If patients were unwilling or unable to complete the testing procedures, had systolic dysfunction (meaning left ventricular ejection fraction was less than $50 \%$ ), were marked with symptoms or signs of congestive HF, had cardiac murmur revealed by a physical examination, suffered significant valvular stenosis or regurgitation displayed in an echocardiography, displayed ischemic HD evidence, showed any documented bradyarrhythmia, tachyarrhythmia, or aborted cardiac death, metabolic disorders including diabetes mellitus, impairment of renal function (defined as serum creatinine greater than $1.2 \mathrm{mg} / \mathrm{dl}$ ), or a history of malignancy they were excluded from the study [12]. After all exclusion factors were considered, 79 patients completed the study. 
The following information is a representation of the findings of polysomnography and echocardiogram testing completed by the participants in the study conducted by Chen et. al. Of the 79 patients, 65 ended up in the moderate-to-severe OSA category and the remaining 14 were in the mild or no OSA category [12]. Similar to many studies, moderate to severe OSA was defined as 15 or more apneas an hour and less than 15 for mild or no OSA on the apnea hypopnea index (AHI). One goal of the study was to investigate further and examine the impact of OSA on cardiac function and remodeling (changes in the heart due to damage). As the data suggests, there were multiple findings that show a significant difference important to the function of the heart. Some of these findings included that left atrial size, mitral A-wave velocity, and left ventricular end-diastolic volume were significantly larger in patients with moderate to severe OSA than in patients with mild or no OSA [12].

Researchers found that patients with moderate-to-severe OSA had issues with the left ventricle filling properly between contractions which is noted by the E/A ratio. E/A being the ratio of early to late ventricular filling velocities. It is also important to note that this finding was independent of the presence of hypertension in the patients. There were also notable differences in the effects of OSA on the patients in rapid eye movement (REM) sleep and in non-rapid eye movement (NREM) sleep. It was found that the aortic root size, E/A ratio, left ventricular end-diastolic volume, left ventricular end-systolic volume, and left ventricular stroke volume were strongly associated with AHI in REM sleep but not in NREM sleep [12]. When looking at patients with SA without clinical symptoms of congestive heart failure, this finding suggests that high $\mathrm{AHI}$ 
in REM sleep could be a potential factor for predicting ventricular dysfunction and changes in cardiovascular structure.

Chen, Su, et. al. conducted one of the few studies that put patients through an overnight polysomnography study and an echocardiography. In most cases, studies only used ESS and Berlin Questionnaires. Although the ESS and the Berlin Questionnaire are relatively accurate (70\% to $80 \%)$, an overnight polysomnography study and echocardiography are more accurate testing methods. Due to the accurate testing methods used in this study, the information that was found is very important and in alignment with many of the findings in other studies. This study confirmed that SA can have detrimental impacts on the proper function of the heart. 


\section{Central control Issues in Sleep Apnea (Sleep Disordered Breathing)}

A more in-depth look at CSA is important in order to try and truly understand what it is and how it effects the heart. In order to better understand CSA it is important to more clearly define it as well as take a look at some of the current research being done. When an individual has CSA, there is an imbalance in the brain's respiratory control centers when the individual is sleeping. When this happens, the mechanisms that monitor the levels of carbon dioxide and oxygen in the blood are reacting too slowly. In simpler terms, the brain is not sending the correct signals to the muscles that control breathing [18]. Once the systems are not reacting fast enough, the body struggles to maintain an even respiratory rate. This begins to cause episodes of apnea where the individual is not breathing and/or hyper apnea (starting to breathe more rapidly). In addition, individuals with CSA can stop breathing and the body will seem as if it is completely at rest. The body is not struggling to try and take a breath as it is in a relaxed state. No movement of the diaphragm or chest cavity can be seen. Now that a better understanding of CSA has been established, it is time to see some of the issues that come along with it.

Due to the complexity of CSA it is possibly even more important than OSA, although it is not as prevalent as OSA. In fact, CSA is only diagnosed in about $5 \%$ of individuals that undergo a sleep study [14]. Similar to OSA, CSA is more often found in men than in women. Although some of the effects of CSA are similar to OSA, it is quite different in it nature. CSA deals more with the brain and proper function of the feedback mechanisms that are responsible for respiratory function. CSA is characterized by reduced respiratory regulation while an individual is sleeping. The outcome of this is 
that one has decreased or absent ventilation and disturbed gas exchange [13]. If the systems are not functioning properly, CSA can cause disturbances in an individual's breathing. An individual may stop breathing for 10-30 seconds and this may even occur for as long as 2 minutes in some cases. During this time, an individual is obviously not taking in any oxygen, negatively impacting oxygen saturation levels.

CompSAS, similar to OSA, is far more likely to occur in men than in women. The development of central or complex events is more complicated than OSA and in many cases can lead to failure of CPAP treatment. Basically, if an individual has CPAP failure it means the person did not respond correctly to the treatment (typically due to the CPAP treatment not being set correctly). Therefore, it is very important that when an individual undergoes treatment everything is set very specifically to that individual.

Gupta and Knapp (2014) found that having insomnia and SA show poorer adherence to CPAP use. Having SA can lead to hyperactivity of the hypothalamicpituitary-adrenal axis (HPA) and sympathetic nervous system (SNS). These issues can cause hyper arousal or insomnia, leading to an increase in cortisol levels and eventually leading to other diseases that are associated with HPA such as cardiovascular disease. If the theory is correct, then improvements with cardiovascular symptoms should be seen when the OSA is treated. According to the American Sleep Apnea Association, when SA is untreated it can have serious consequences such as an increase in blood pressure, HD, sleepiness and stroke [3]. Insomnia too can be problematic when not treated and over time it can cause stress, anxiety and other damaging effects to the body and heart. Over time, stress and anxiety can lead to some of the damaging effects that are discussed throughout this paper. 


\section{Complex/Mixed SA}

CompSAS is when an individual has a combination of OSA and CSA. Similar to other SA conditions, CompSAS is more common in males than in females. For the most part CompSAS can develop when an individual has OSA. This usually occurs due to having severe OSA or improper treatment of OSA. Some studies have even shown that CompSAS typically emerges after the OSA has disappeared with CPAP treatment [13]. Due to the connection between OSA and CompSAS, it is important to find a treatment that will treat all forms of SA. According to Khan and Franco CompSAS is currently defined as having central events comprising of more than half of the residual sleep disordered breathing events. CompSAS can also lead to a periodic breathing pattern which on positive airway pressure therapy becomes predominant and disruptive and the central apnea index (CAI must be $>5$ events/hour. This review article also suggested that it is unsure whether individuals with central apneas associated with narcotics or Cheyne-Stokes breathing should be included due to systolic heart failure.

In an effort to better define the population Khan \& Franco (2014) left out the individuals with central apneas associated with Cheyne-Stokes breathing and narcotics. Differentiating between CompSAS, OSA, and CSA is important in order to characterize the pathophysiology of CompSAS. It is also important to understand CompSAS so that better treatment and management strategies can be established. There are many factors believed to influence CompSAS, but more research needs to be conducted to further explore these factors. Interaction of upper airway obstruction, unstable central ventilator control factors, and certain host conditions are considered to be the key factors [13]. It is also thought that CompSAS typically occurs when an individual is in an 
unstable sleep state. One clinical finding noted that individuals with CompSAS reported more CPAP removal during sleep [13].

There is not a great deal of information available regarding the effects of CompSAS. However, since CompSAS is a mixture of OSA and CSA, one could likely assume the effects of CompSAS would be a mixture of OSA and CSA effects. Regarding treating CompSAS, there are a number of treatment methods. Some of these methods include CPAP, Adaptive Servoventilation (ASV), and CO2 administration. It has been found that ASV was more reliable and effective for treating CompSAS than CPAP. In one study, $66 \%$ of participants experienced successful treatment with CPAP and $90 \%$ of the patients in the same study found treatment success with ASV [15]. Although one method seems to have a better rate of success, both of these methods seemed to produce similar results.

Improper CPAP treatment seems to be one of the biggest contributors to CompSAS. Many studies found that over treating CPAP is typically what leads to CompSAS. Therefore, when treating CompSAS and OSA (since OSA often leads to CompSAS), it is important to understand the proper amount of pressure needed for each individual and to not exceed that specified amount in order to avoid the development of CompSAS. 


\section{Severity of SA}

The concept and importance of severity is critical to explore. It is important to discuss the concept of severity due to the relatively linear relationship it has with cardiovascular problems as SA events increase. As discussed earlier, individuals that have more severe SA episodes per hour seem to have greater risk for cardiovascular problems compared to those with little or no SA. In other words, severity plays a large role in the outcome of an individual that has one or more forms of SA.

Valenza, Baranchuk, et. al. found that there was a significant risk for atrial fibrillation (AF) and stroke in patients with SA. In addition, research showed that as the severity of SA increased, the likelihood of experiencing one of these two heart complications also increased. Hypertension was the most common cardiovascular condition amongst these patients. The prevalence of hypertension and absolute systolic blood pressure was higher in the patients with severe SA (diagnosed according to CHADS2 score). According to the Sleep Heart Health Study, men that had moderate to more severe SA were close to three times more likely to have a stroke than those with mild SA or no SA at all. The results were similar for women however the increased risk was only significant with severe levels of SA. This same study showed that those with severe SA have a four-fold increased risk of AF when compared to those in the control groups without SA [7]. Javadi and Jalilolghadr's (2014) studies demonstrated the intensity of coronary artery disease increased in patients with OSA. Along with this subjects showed a significant correlation between the intensity of OSA and excessive daytime sleepiness. 


\section{Diagnosing SA}

The standard method for diagnosing OSA involves an overnight polysomnography (PSG). The PSG includes electroencephalogram, electromyogram, electrocardiogram (ECG), oximetry, oronasal air flow, and respiratory efforts [7]. Using these methods, it is possible to find the existence and intensity of SA. Knowing the intensity of the SA is very important considering there seems to be a direct or linear correlation between the severity of SA and the impact on an individual. Unfortunately, PSG is a very costly method for screening for SA and it would be impossible to use this method for screening everyone. The most common methods for screening individuals for sleep apnea are the Berlin Questionnaire and Epworth Sleepiness Scale (ESS). One of the main reasons for this is the fact that the cost is much lower compared to PSG testing. A survey done at 20 teaching hospitals involving OSA patients showed the results of ESS compared with PSG findings for assessing sleep apnea [7]. They found sensitivity and specificity of the Berlin questionnaire for assessing OSA were found to be $85 \%$ and $77 \%$ respectively [7]. These methods are very good indicators for diagnosing OSA in patients. Once a patient is diagnosed in this format, the individual can be referred to a specialist to undergo further assessment. 


\section{Future SA Studies}

A study done on the Sleep-Disordered Breathing in Heart Failure (SCHLA-HF) registry could potentially lead to a better understanding and management of cardiovascular disease. The registry is important in helping explore the association between heart disease and SA.

All German sleep laboratories were invited to participate in the registry. Due to constraints including lack of necessary infrastructure, many laboratories were asked to contact cardiologists in their referral area to build networks. The networks would be built between cardiology and sleep medicine. Networks were built in order to allow the registry and the SERVE-HF study to proceed. The participating cardiologists were then asked to enroll all heart failure patients that fulfilled the criteria in order to build the registry.

There were one hundred and thirty eight centers in Germany testing patients with chronic HF-REF for eligibility for enrollment in the registry. Of the 138 centers, 91 were cardiology practices and 47 were hospital cardiology departments. After patients were enrolled in the registry, they were screened for SA. If patients had 15 or more apneas or hypopneas an hour, they were considered to have symptoms of SA and were referred to one of the 66 cooperating sleep clinics. At the clinic, patients participated in a polysomnography (PSG), a definite diagnosis was made, and suggested treatment was documented. This registry spanned from October 2007 to May 2013.

According to Woehrle, Oldenburg, et. al, in order to be included in the registry the patients were screened and needed to meet the following criteria: chronic HF diagnosed 
and treated according to the European Society of Cardiology (ESC) guidelines [23] $\geq 12$ weeks prior to enrolment; moderate-to-severe left ventricular systolic dysfunction (left ventricular ejection fraction $[\mathrm{LVEF}] \leq 45 \%$ by an imaging method such as echocardiography, radionuclide angiography, left ventriculography or cardiac magnetic resonance imaging) documented $<12$ weeks before enrollment; New York Heart Association (NYHA) class III or IV at the time of inclusion or NYHA class II with $\geq 1$ hospitalization for HF in the last 12 months. The patients also had to be able to fully understand the study information and give an informed consent. There was an additional list of reasons that patients could not be included in the registry to better control the results. 


\section{$\underline{\text { Conclusion }}$}

Each day, more research is conducted on SA and HD. As the amount of research conducted increases, the idea that SA is potentially an independent risk factor for HF seems to gain strength. More specifically, research seems to indicate that OSA and CSA are risk factors or causes of HF, CSA being the stronger risk factor. Knowing that the preponderance of evidence is pointing to a direct correlation between the two, it is very important to continue to study this topic until the potential correlation is fully investigated. Since HF is one of the leading causes of death in the U.S. in both men and women, the need to further study SA and its effects on HF is apparent. If the current findings indicate that there is a strong correlation between SA and HF, education and intervention could drastically decrease the number of individuals dying from HF. In addition, we need to study SA to find the most effective treatment to increase the quality and longevity of an individual's life. It would also be beneficial to further test the accuracy of the Berlin Questionnaire and ESS to have a simple method for diagnosing SA. Even though these tests are not $100 \%$ effective for diagnosing SA in individuals, they are still relatively accurate and inexpensive testing methods consisting of a simple scale that patients could fill out on a regular basis at the doctor's office. These tests could be a way to catch SA in many individuals that would otherwise go undiagnosed. If individuals are found to be a candidate for having SA, they could then be referred to a clinic for proper polysomnography testing. In the future this kind of testing could greatly reduce SA problems across the population. Below in Figure 1 is an example of Berlin Questionnaire and in figure 2 is the ESS to show the simplicity of the tests and the contents. 
Figure 1. Berlin Questionnaire

Name:

D.O.B.

Age:

Height (m):

weight $(\mathrm{kg})$ :

$\operatorname{Neck}(\mathrm{cm})$ :

IMPORTANT: Please give only one answer per question by typing Yes in the relevant boxes

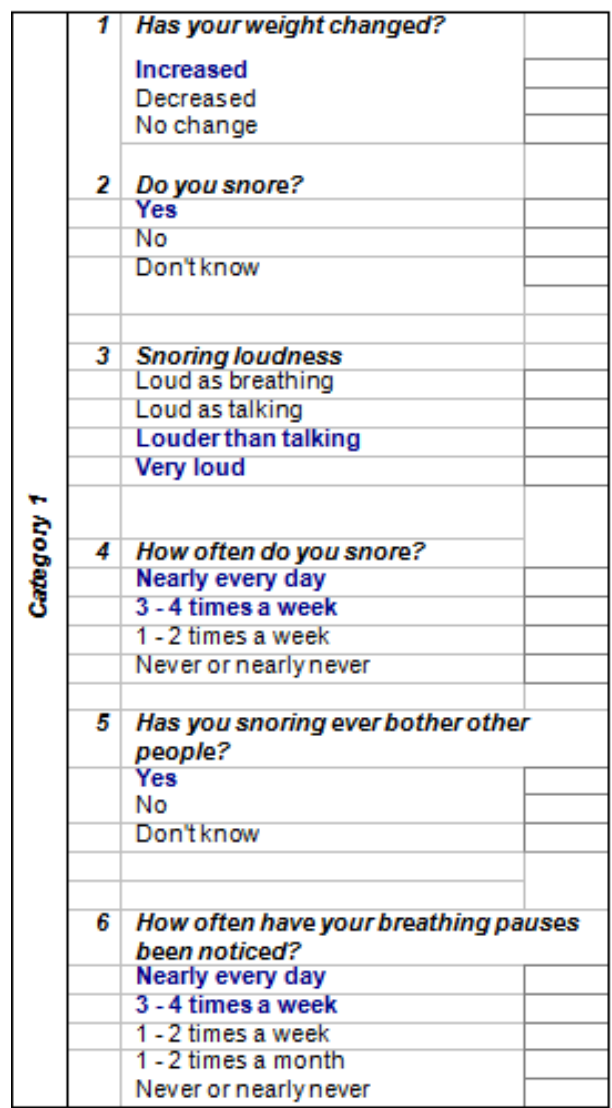

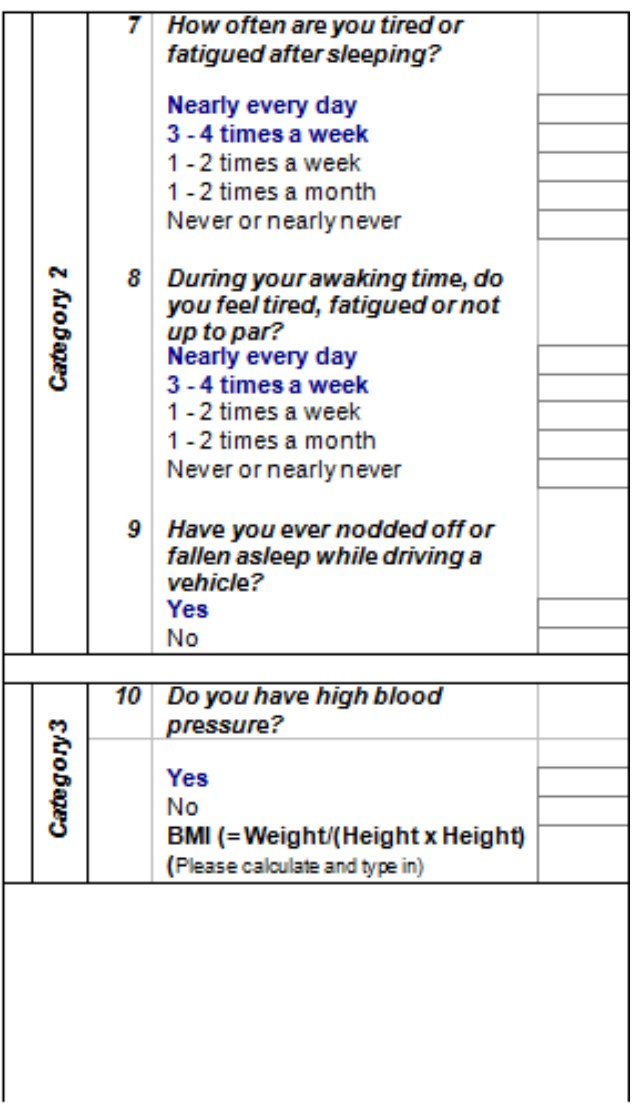


Figure 2. Epworth Sleepiness Scale (ESS)

EPWORTH SLEEPINESS SCALE (ESS)

Patient self assessment questionnaire

Please fill in the following questionnaire by filling in a number in the box by each situation

In each situation, please try and estimate the chance of you dozing

$\begin{array}{rr}\text { Would NEVER doze } & 0 \\ \text { SLIGHT chance of dozing } & 1 \\ \text { MODERATE chance of dozing } & 2 \\ \text { HIGH chance of dozing } & 3\end{array}$

\begin{tabular}{|l|l|}
\hline Sitting reading a book & \\
\hline Watching television & \\
\hline Sitting inactive in a publicplace e.g. in a meeting & \\
\hline Lying downto rest in the afternoon & \\
\hline Sitting talking to someone & \\
\hline Sitting quietly after a lunch without alcohol & \\
\hline In a car, stoppedintrafficor at lights & \\
\hline In a car, as a passenger for an hour & $\begin{array}{l}\text { Epworth Sleepiness Scale (ESS) TOTAL } \\
\text { (Please add up results typed above and } \\
\text { type result in the grey box) }\end{array}$ \\
\hline
\end{tabular}

There are so many negative effects on the heart that studies are linking to OSA.

These effects are on the autonomic nervous function of the heart as well as many physical effects. Different studies have found problems with hypertension, LV hypertrophy, and BP issues. Some studies have even shown that OSA may be a risk factor for the prevalence of medical conditions such as type 2 diabetes and headaches [11]. With all of these issues being strongly linked to SA, it is hard to deny the need for better and proper diagnosis. Even the one of the simplest effects of SA, daytime sleepiness, can be extremely deadly when it comes daily activities such as driving. Some studies have even shown SA problems in young children. Although most children 
outgrow the SA problems, some children do not. Since the potential negative effects that SA has on the heart and cardiovascular system are great, it is hard to deny the need for better diagnosis and treatment. Research has uncovered many of the signs of SA and clinicians need to educate the general public of these signs and symptoms. Some of these signs of SA include snoring, pauses in breathing, feeling excessively sleepy during the day, gasping or choking leading to awakening, a large neck, and obesity. If treatment of SA can potentially lessen the damaging effects on the cardiovascular system this could make great strides in avoiding unnecessary surgeries. In conclusion, better diagnosis methods, better treatment, and better education to the public about the potential correlation between SA and HF would lead to a better future. 


\section{Special Terminology}

CHADS2 Score: The CHADS2 score is a clinical prediction rule for estimating the risk of stroke in patients with non-rheumatic atrial fibrillation (AF).

Cheyne-Stokes Respiration: This is an abnormal pattern of breathing also known as periodic respiration. Respiration often cycles deep then shallower and often eventually leads to an apnea. 


\section{$\underline{\text { References }}$}

1. Kendzerska T, Gershon AS, Hawker G, Leung RS, Tomlinson G (2014) Obstructive Sleep Apnea and Risk of Cardiovascular Events and All-Cause Mortality: A Decade-Long Historical Cohort Study. PLoS Med 11(2): e1001599. doi:10.1371/journal.pmed.1001599

2. ValenzaMC, et al, Prevalence of risk factors for atrial fibrillation and stroke among 1210 patients with sleep disordered breathing, Int J Cardiol (2014), http://dx.doi.org/10.1016/j.ijcard.2014.03.156

3. Gupta MA, Knapp K (2014) Cardiovascular and Psychiatric Morbidity in Obstructive Sleep Apnea (OSA) with Insomnia (Sleep Apnea Plus) versus Obstructive Sleep Apnea without Insomnia: A Case-Control Study from a Nationally Representative US Sample. PLoS ONE 9(3): e90021. doi:10.1371/journal.pone.0090021

4. Aronson D, Nakhleh M, Zeidan-Shwiri T, Mutlak M, Lavie P, et al. (2014) Clinical Implications of Sleep Disordered Breathing in Acute Myocardial Infarction. PLoS ONE 9(2): e88878. doi:10.1371/journal.pone.0088878

5. Nieto F, Young TB, Lind BK, et al. Association of Sleep-Disordered Breathing, Sleep Apnea, and Hypertension in a Large Community-Based Study. JAMA. 2000;283(14):1829-1836. doi:10.1001/jama.283.14.1829.

6. F. Javier Nieto, Paul E. Peppard, and Terry B. Young, "Sleep Disordered Breathing and Metabolic Syndrome," WMJ. 2009 August ; 108(5): 263-265.

7. Hamid Reza Javadi, Shabnam Jalilolghadr, Zohreh Yazdi, and Zeinab Rezaie Majd, "Correlation between Obstructive Sleep Apnea Syndrome and Cardiac 
Disease Severity," Cardiovascular Psychiatry and Neurology, vol. 2014, Article ID 631380, 5 pages, 2014. doi:10.1155/2014/631380

8. Woehrle et al.: Determining the prevalence and predictors of sleep disordered breathing in patients with chronic heart failure: rationale and design of the SCHLA-HF registry. BMC Cardiovascular Disorders 2014 14:46.

doi:10.1186/1471-2261-14-46

9. Akiko Noda, Seiko Miyata, and Yoshinari Yasuda, "Therapeutic Strategies for Sleep Apnea in Hypertension and Heart Failure," Pulmonary Medicine, vol. 2013, Article ID 814169, 5 pages, 2013. doi:10.1155/2013/814169

10. Grimm W, Koehler U. Cardiac Arrhythmias and Sleep-Disordered Breathing in Patients with Heart Failure. International Journal of Molecular Sciences. 2014; 15(10):18693-18705.

11. Hamid Reza Javadi, Shabnam Jalilolghadr, Zohreh Yazdi, and Zeinab Rezaie Majd, "Correlation between Obstructive Sleep Apnea Syndrome and Cardiac Disease Severity," Cardiovascular Psychiatry and Neurology, vol. 2014, Article ID 631380, 5 pages, 2014. doi:10.1155/2014/631380

12. Chen YL, Su MC, Liu WH, Wang CC, Lin MC,Chen MC. Influence and Predicting Variables of Obstructive Sleep Apnea on Cardiac Function and Remodeling in Patients without Congestive Heart Failure. J Clin Sleep Med 2014; 10: 57-64.

13. Muhammad Talha Khan and Rose Amy Franco, "Complex Sleep Apnea Syndrome," Sleep Disorders, vol. 2014, Article ID 798487, 6 pages, 2014. doi:10.1155/2014/798487 
14. American Sleep Apnea Association | Sleep Apnea. (n.d.). Retrieved from http://www.sleepapnea.org/learn/sleep-apnea.html

15. CDC - DHDSP - Heart Disease Facts. (n.d.). Retrieved from http://www.cdc.gov/heartdisease/facts.htm

16. Young, P.E. Peppark, and D.J. Gottliech, "Epidemiology of obstructive sleep apnea: a population health perspective," The American Journal of Respiratory and Critical Care Medicine, Vol, 165, no.9 pp. 1217-1239, 2002

17. Sleep Heart Health Study. "Methods for obtaining and analyzing unattended polysomnography data for a multicenter study." Sleep Heart Health Research Group. Redline S, Sanders MH, Lind BK, Quan SF, Iber C, Gottlieb DJ, Bonekat WH, Rapoport DM, Smith PL, Kiley JP. Sleep. 1998 Nov 1;21(7):759-67.

18. Central sleep apnea Definition - Diseases and Conditions - Mayo Clinic. (n.d.). Retrieved from http://www.mayoclinic.org/diseases-conditions/central-sleepapnea/basics/definition/con-20030485

19. Amereican heart association Retrieved from http://www.heart.org August, 152015

20. National Institutes of Health Retrieved from http://www.nhlbi.nih.gov September, 132015 